http://jmscr.igmpublication.org/home/ ISSN (e)-2347-176x ISSN (p) 2455-0450 crossref DOI: https://dx.doi.org/10.18535/jmscr/v7i12.92

\title{
Is a Scoring System Valid for Detecting Common Bile Duct Stones in Gallstone Pancreatitis
}

Authors

\author{
Dr Sudhir M. ${ }^{1}$, Dr Shravan Kumar K R ${ }^{2 *}$, Dr Amrit Preetam Panda ${ }^{3}$, Dr Deepak Lal ${ }^{4}$ \\ ${ }^{1}$ MBBS, MS, Professor of General Surgery \\ ${ }^{2,3,4}$ MBBS, Postgraduate in General Surgery, Kempegowda Institute of Medical Sciences, Bengaluru \\ *Corresponding Author \\ Dr Shravan Kumar K R
}

Room No 304, Kimshospital Residents' Quarters, Kempegowda Institute of Medical Sciences and Hospital,

V V Puram, Bengaluru-560004

\begin{abstract}
Background: The passage of gallstones from the gallbladder through the common bile duct (CBD) into the duodenum has been implicated in the pathophysiology of acute Gall stone pancreatitis. Most stones in the $C B D$ pass spontaneously; however, retained CBD stones cause persistent biliary obstruction and morbidity increase when obstruction is present for $>48$ hours. The scoring system and protocol proposed by a 2009 study simplifies the diagnosis and management of retained CBD stones in gallstone pancreatitis by using 5 variables obtained at admission. Special consideration must be given in the setting of a persistent CBD stone because it changes the management of the case; in addition to cholecystectomy, the CBD stone must be removed.
\end{abstract}

Methods: 40 patients who were admitted with Gallstone pancreatitis in kempegowda institute of medical sciences, Bangalore from December 2017 to August 2019 under department of general surgery. Patients were evaluated for presence of common bile duct (CBD) calculi using USG, MRCP, CECT and were appropriately referred for ERCP (Endoscopic retrograde cholangio Pancreatography) for extraction of CBD calculi.

Patients are given 1 of 6 possible scores (0-5). Initial admission laboratory values and ultrasonography results are used.

One point assigned for each of the following;

a) CBD size - > =9 mm, (CBD-Common Bile Duct)

b) $G G T->=350$ U/L, (Gamma glutamyl transferase)

c) $A P->=250 \mathrm{U} / \mathrm{L}$, (Alkaline Phosphatase)

d) $T B->=3 \mathrm{mg} / \mathrm{dL}$, (Total Bilirubin)

e) $D B->=2 \mathrm{mg} / \mathrm{dl}$ (Direct Bilirubin)

Proposed Score Based Treatment
a) 0- LAPAROSCOPIC CHOLECYSTECTOMY
b) 1- LAPAROSCOPIC CHOLECYSTECTOMY
c) 2- MRCP ASSESSMENT + LAPAROSCOPIC CHOLECYSTECTOMY
d) 3 and 4 -MRCP ASSESSMENT + LAPAROSCOPIC CHOLECYSTECTOMY
e) 5- ERCP 
Results: Total study population consisted of 40 patients of which 13 patients had CBD stones and 27 patients didn't have any CBD stones. .Majority of the study population consisted of Females(65\%) and major population group was aged between 51 -60 age group (including males and females 35\%). Majority of the population group underwent laparoscopic cholecystectomy (36 patients) while 2 patients underwent Open $C B D$ exploration and 2 patients underwent ERCP only during the study period. According to our scoring system overall negative predictive value (NPV) of the scoring system was $83.3 \%$ while overall positive predictive value (PPV) was $56.3 \%$ for the scoring system of which score 5 had $100 \%$ PPV. The overall accuracy of the scoring system was $72.5 \%$.

Conclusion: The overall accuracy of the study is $72.5 \%$ using this scoring system. we propose the following for patients: those with score 0 or 1 undergo LC with follow up To rule out biliary obstruction or recurrent pancreatitis post-operatively; score 2,3 or 4 undergo MRCP assessment and score 5 should go directly to ERCP. Every patient is advised LC as a definitive treatment to prevent recurrence of Gall stone. Pancreatitis in same admission or for interval cholecystectomy. The proposed scoring system helps eliminate negative ERCPs and complications associated with ERCPS.

Keywords: Common bile duct stones, Gall stone Pancreatitis, Retained CBD stones, Laparoscopic cholecystectomy.

\section{Introduction}

Acute pancreatitis is defined as an acute condition presenting with abdominal pain, a threefold or greater rise in the serum levels of the pancreatic enzymes amylase or lipase, and or characteristic findings of pancreatic inflammation on contrast- enhanced CT.

Gallstones are the most common cause of acute pancreatitis, accounting for $44-54 \%$ of cases. ${ }^{2,3}$ The overall incidence of AP in patients with symptomatic gallstone disease is $3 \%$ to $8 \%$. It is seen more frequently in women between 50 and 70 years of age. ${ }^{4}$

Gallstone disease is one of the most common problems affecting the digestive tract with a prevalence of 11 to $36 \%$. Large screening ultrasound studies have shown that gallstones are present in $5 \%$ to $20 \%$ of adults ${ }^{6,7,8}$. The prevalence ranges widely across ethnicities and is approximately twice as high in women as in men $^{9,10,11}$.

Potential complications of gallstone pancreatitis include sepsis, cholangitis, necrotizing pancreatitis, hemorrhagic pancreatitis, and death. The mechanism of gallstone pancreatitis is not fully understood, but is believed to be owing to stone migration into the common bile duct (CBD), resulting in bile reflux into the pancreatic duct and triggering trypsin activation and autodigestion of the pancreas ${ }^{12,13}$. Most stones in the CBD pass spontaneously; however, retained CBD stones cause persistent biliary obstruction and morbidity increase when obstruction is present for $>48$ hours. Occult Biliary microlithiasis may be the etiology in a majority of cases of idiopathic acute pancreatitis $^{14}$.

\section{Aims and Objectives}

To validate the proposed scoring system and management strategies in retained CBD stones post gall stone pancreatitis.

\section{Methods}

After clinical approval of Institutional Ethical Clearance Committee and informed written consent, 40 patients presenting with gall stone pancreatitis at KIMS Hospital, Bengaluru were selected for the study. The study was conducted from December 2017 to July 2019 in the Department of Surgery of KIMS Hospital, Bengaluru. The study was conducted using the Hospital Observational Study model.

We used the same 5 quantitative variables and their cut offs as determined by the original study to determine the score for each patient enrolled in the study. Patients were given 1 of 6 possible scores (0-5). Initial admission laboratory values and ultrasonography results were used.

One point was assigned for each of the following: CBD size $>/=9 \mathrm{~mm}$, GGT $>/=350 \mathrm{U} / \mathrm{L}$, ALP $>/=$ 
$250 \mathrm{U} / \mathrm{L}, \mathrm{TB}>/=3 \mathrm{mg} / \mathrm{dL}$, and $\mathrm{DB}>/=2 \mathrm{mg} / \mathrm{dL}$. As Intraoperative Cholangiogram was not being used routinely in our setup, patients with low suspicion of having retained CBD stones were closely followed up to look for recurrence of pancreatitis or biliary obstruction in case of any undetected retained distal CBD stones. If the patients did present with any of the features mentioned above and USG suggested obvious biliary obstruction, they were sent for ERCP straightaway. However, in case of no known cause of biliary obstruction, they were subjected to MRCP first.

Score 0 - LC with follow up for 3 months

Score 1 - LC with follow up for 3 months Score 2,3,4 - MRCP assessment with LC Score 5 - ERCP with LC

\section{Protocol of Study}

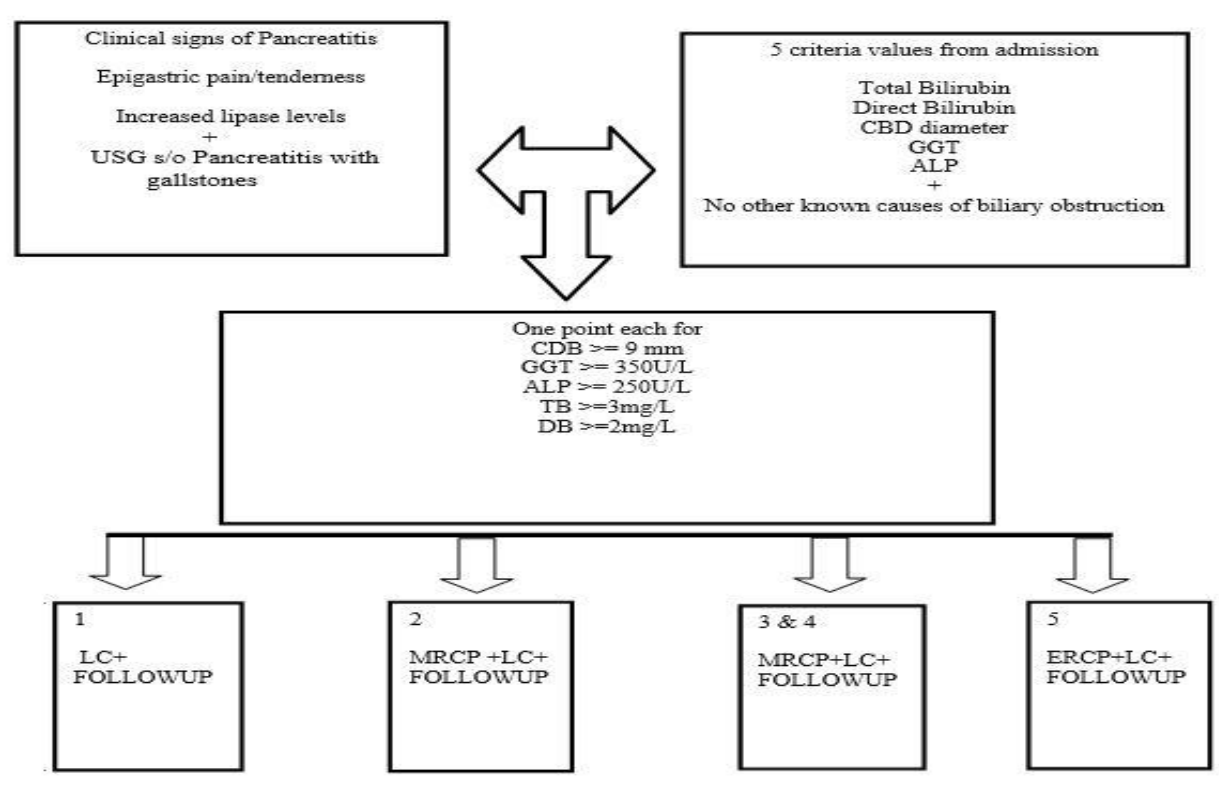

\section{Results}

Statistical Package for Social Sciences [SPSS] for Windows, Version 22.0. Released 2013. Armonk, NY: IBM Corp., was used to perform statistical analyses. Chi Square Test was used to perform age and gender wise comparison of prevalence of CBD stone, distribution of scores based on the presence of $\mathrm{CBD}$ stone, various treatment modalities proposed based on newly devised scoring system among study patients. Mann Whitney $U$ test was used to compare the mean values of different study variables based on the Presence of CBD Stone. Sensitivity \& Specificity Analysis was performed for estimating the accuracy of new scoring system as compared to USG Scanning in detecting CBD Stone. The level of significance [P-Value] was set at $\mathrm{P}<0.05$.

Table showing age and gender wise prevalence of cbd stone in the study

\begin{tabular}{|l|c|c|c|}
\hline \multicolumn{4}{|c|}{ Age and Gender distribution among study Patients } \\
\hline Variable & Category & $\mathrm{n}$ & $\%$ \\
\hline Age & $21-30$ years & 7 & $17.5 \%$ \\
\cline { 2 - 4 } & $31-40$ years & 5 & $12.5 \%$ \\
\cline { 2 - 4 } & 41-50 years & 8 & $20.0 \%$ \\
\cline { 2 - 4 } & 51-60 years & 14 & $35.0 \%$ \\
\cline { 2 - 4 } & $>60$ years & 6 & $15.0 \%$ \\
\hline Sex & Males & 14 & $35.0 \%$ \\
\cline { 2 - 4 } & Females & 26 & $65.0 \%$ \\
\hline
\end{tabular}


Table showing comparison of mean values using mann whitney u test

Comparison of mean values of different study variables based on the Presence of CBD Stone using Mann Whitney U test

\begin{tabular}{|c|c|c|c|c|c|c|}
\hline Variables & CBD STONE & $\mathrm{N}$ & Mean & SD & Mean Diff & P-Value \\
\hline \multirow[t]{2}{*}{ S Amylase } & Yes & 13 & 1105.56 & 1119.73 & \multirow{2}{*}{67.00} & \multirow{2}{*}{0.85} \\
\hline & No & 27 & 1038.56 & 781.01 & & \\
\hline \multirow[t]{2}{*}{ S Lipase } & Yes & 13 & 462.31 & 292.43 & \multirow{2}{*}{11.46} & \multirow{2}{*}{0.59} \\
\hline & No & 27 & 450.85 & 368.54 & & \\
\hline \multirow[t]{2}{*}{ USG (CBD) } & Yes & 13 & 10.09 & 4.20 & \multirow{2}{*}{5.12} & \multirow{2}{*}{$<0.001 *$} \\
\hline & No & 27 & 4.97 & 1.90 & & \\
\hline \multirow[t]{2}{*}{ CBD SIZE } & Yes & 13 & 10.47 & 3.81 & \multirow{2}{*}{5.50} & \multirow{2}{*}{$<0.001 *$} \\
\hline & No & 27 & 4.97 & 1.90 & & \\
\hline \multirow[t]{2}{*}{ GGT } & Yes & 13 & 299.23 & 258.33 & \multirow{2}{*}{55.90} & \multirow{2}{*}{0.57} \\
\hline & No & 27 & 243.33 & 159.81 & & \\
\hline \multirow[t]{2}{*}{ ALP } & Yes & 13 & 209.85 & 89.72 & \multirow{2}{*}{51.78} & \multirow{2}{*}{$0.02 *$} \\
\hline & No & 27 & 158.07 & 113.08 & & \\
\hline \multirow[t]{2}{*}{ Total Bilirubin } & Yes & 13 & 3.53 & 2.54 & \multirow{2}{*}{1.42} & \multirow{2}{*}{$0.04 *$} \\
\hline & No & 27 & 2.12 & 2.17 & & \\
\hline \multirow[t]{2}{*}{ Direct Bilirubin } & Yes & 13 & 2.97 & 2.36 & \multirow{2}{*}{1.46} & \multirow{2}{*}{$0.04 *$} \\
\hline & No & 27 & 1.51 & 1.90 & & \\
\hline
\end{tabular}

Table showing scores based on presence of cbd stone among patients using chi square test

\begin{tabular}{|c|c|c|c|c|c|c|c|}
\hline \multirow[b]{2}{*}{ Variable } & \multirow[b]{2}{*}{ Category } & \multicolumn{2}{|r|}{ Yes } & \multicolumn{2}{|r|}{ No } & \multirow[b]{2}{*}{$\chi^{2}$ Value } & \multirow[b]{2}{*}{ P-Value } \\
\hline & & $\mathrm{n}$ & $\%$ & $\mathrm{n}$ & $\%$ & & \\
\hline \multirow[t]{6}{*}{ Scores } & Score 0 & 2 & $11.1 \%$ & 16 & $88.9 \%$ & \multirow{6}{*}{12.143} & \multirow{6}{*}{$0.03 *$} \\
\hline & Score 1 & 2 & $33.3 \%$ & 4 & $66.7 \%$ & & \\
\hline & Score 2 & 3 & $50.0 \%$ & 3 & $50.0 \%$ & & \\
\hline & Score 3 & 3 & $50.0 \%$ & 3 & $50.0 \%$ & & \\
\hline & Score 4 & 0 & $0.0 \%$ & 1 & $100.0 \%$ & & \\
\hline & Score 5 & 3 & $100.0 \%$ & 0 & $0.0 \%$ & & \\
\hline
\end{tabular}

Table showing sensitivity and specificity of new scoring system compared to usg in detecting cbd stone

Sensitivity \& Specificity Analysis for estimating the accuracy of new scoring system as compared to USG

Scanning in detecting CBD Stone

\begin{tabular}{|l|c|c|c|}
\hline \multirow{2}{*}{ Scoring System } & \multicolumn{2}{|c|}{ USG CBD Stone } & \multirow{2}{*}{ Total } \\
\cline { 2 - 3 } & Present & Absent & 16 \\
\hline Present & 9 & 7 & 24 \\
\hline Absent & 4 & 20 & 40 \\
\hline Total & 13 & 27 & 40 \\
\hline
\end{tabular}

\begin{tabular}{|l|c|c|}
\hline & Present & Absent \\
\hline Present & True +ve & False +ve \\
\hline & False & True \\
Absent & -ve & -ve \\
\hline
\end{tabular}

\begin{tabular}{|c|c|c|c|c|}
\hline \multirow{2}{*}{$\begin{array}{l}\text { Diagnostic } \\
\text { accuracy }\end{array}$} & \multirow[b]{2}{*}{$\%$} & \multicolumn{2}{|c|}{$95 \% \mathrm{CI}$} & \\
\hline & & Lower & Upper & \\
\hline Sensitivity & $69.2 \%$ & $38.6 \%$ & $90.9 \%$ & \\
\hline Specificity & $74.1 \%$ & $53.7 \%$ & $88.9 \%$ & \\
\hline PPV & $56.3 \%$ & $38.2 \%$ & $72.8 \%$ & \\
\hline NPV & $83.3 \%$ & $68.2 \%$ & $92.1 \%$ & \\
\hline Accuracy & $72.5 \%$ & $56.1 \%$ & $85.4 \%$ & \\
\hline \multirow{2}{*}{$\begin{array}{ll}\text { Area } & \text { Under } \\
\text { Curve } & \end{array}$} & \multirow[b]{2}{*}{ Std. Error } & \multirow[b]{2}{*}{ P-Value } & \multicolumn{2}{|c|}{$95 \% \mathrm{Cl}$} \\
\hline & & & Lower & Upper \\
\hline 0.72 & 0.09 & $0.03 *$ & 0.54 & 0.89 \\
\hline
\end{tabular}




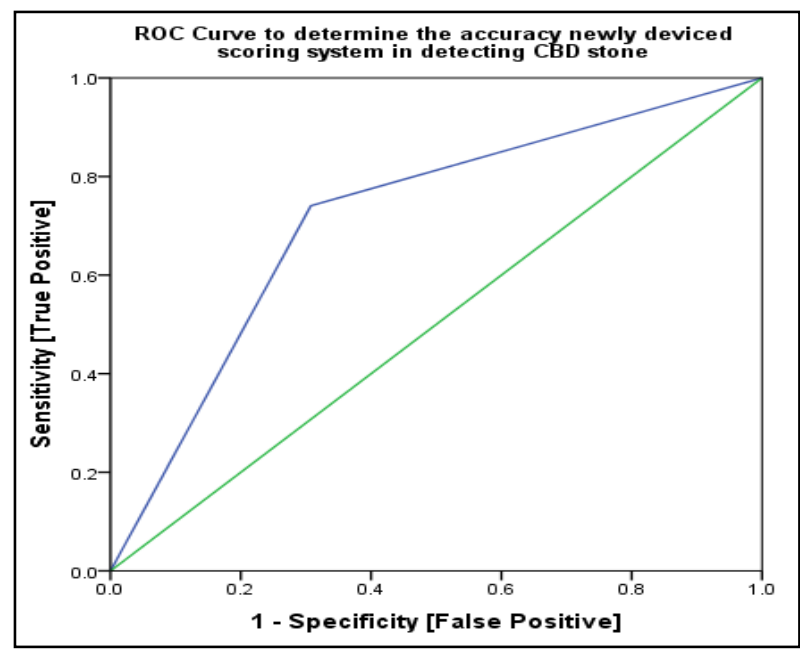

ROC curve to determine the accuracy of new scoring system in detecting CBD stone

\section{Discussion}

The definitive treatment for gallstone pancreatitis is cholecystectomy. However, persistent CBD stones must be addressed. The diagnosis and management of persistent CBD stones in gallstone pancreatitis varies among practitioners. A 2009 paper by Telem et al used retrospective data and multivariate analysis to determine 5 quantitative variables and their cut offs that have a positive predictive value (PPV) for CBD stones ${ }^{15}$ :

1. CBD size $>/=9 \mathrm{~mm}$

2. Gamma glutamyl transferase $(\mathrm{GGT})>/=350$ $\mathrm{U} / \mathrm{L}$

3. Alkaline phosphatase (ALP) $>/=250 \mathrm{U} / \mathrm{L}$

4. Total bilirubin $(\mathrm{TB})>/=3 \mathrm{mg} / \mathrm{dL}$

5. Direct bilirubin (DB) $>/=2 \mathrm{mg} / \mathrm{dL}$.

In a follow-up paper from the same institute by Sherman et al $^{16}$ a scoring system was suggested and validated for use in cases of gallstone induced acute pancreatitis to detect retained CBD stones.
Under the proposed system, at the time of admission, the above 5 criteria were assessed and 1 point was given for the presence of each. Majority of the patients with scores $0-1$ did not have CBD stones, whereas, patients with scores 45 had $92 \%$ risk of persistent CBD stones. The results of this study recommended that patients with a score of 0 undergo laparoscopic cholecystectomy with intraoperative cholangiogram (LCIOC), treatment of patients with scores of 1-3 were to be made on an individual basis, and those with scores of 4 and 5 were to undergo endoscopic retrograde cholangiopancreatography (ERCP) and necessary procedures such as sphincterotomy, stone extraction and stent insertion.

The Department of General Surgery at KIMS Hospital, the study center admits many patients of gallstone pancreatitis. Our prospective data confirms that ALP, GGT, TB, DB, and CBD size at the time of admission are 5 factors that can differentiate between those patients with and without CBD stones. Although AST and ALT were found to be higher among patients with CBD stones, the result was not significant in our study. Our data show that scores of 5 is very accurate $(100 \%)$ in predicting the presence of CBD stone, while score $0(88.9 \%)$ and $1(66.7 \%)$ was able to predict absence of CBD stone respectively. Scores of 1-4 should be used to determine the next step in diagnosing the presence of a CBD stone. We propose the following protocol for patients suffering from gallstone pancreatitis,

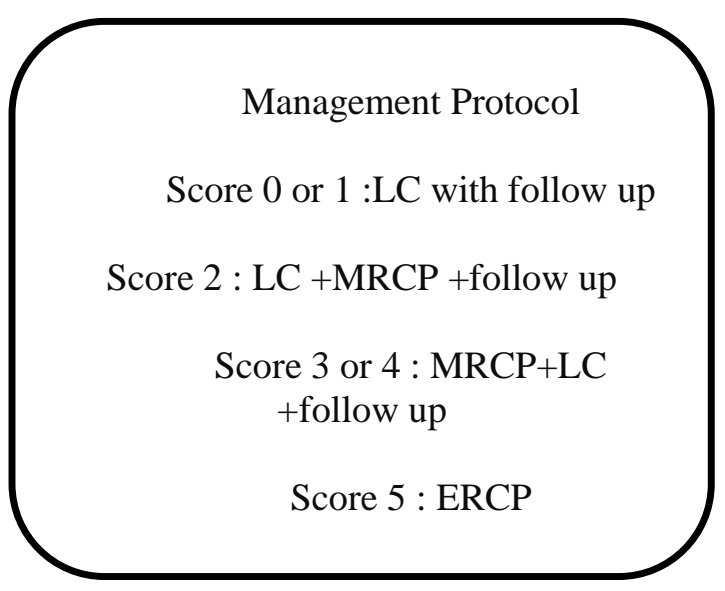


Patients with 0 score should proceed directly to laparoscopic cholecystectomy as an initial treatment, as they have a very low chance of having stones with follow up. There were 18 Patients with score 0 in which $16(88.9 \%)$ didn't have any CBD stones and $2(11.1 \%)$ had CBD stones.

Two Patients in our study with score 1 had retained CBD stone were exception, as one patient had dilated CBD $(9 \mathrm{~mm})$ in USG and other had retained CBD Stone post lap cholecystectomy were sent for ERCP. Patients with score 1 need to undergo MRCP assessment based on treating surgeon preference assessing patients clinical condition and laboratory parameters. Other 4 patients with score 1 were then being followed up postoperatively to look for recurrence of pancreatitis or biliary obstruction in case of any undetected retained distal CBD stones.

Patients with score of 2, 3, 4 were made to undergo MRCP to detect CBD stones, as these patients have a high probability to be having CBD stones. Instead of subjecting the patients to an unnecessary ERCP, which is quite probable in such cases, it is better to evaluate the biliary tree using MRCP first. If MRCP showed a stone, the patient can undergo stone removal either with ERCP or CBD exploration based on surgeon preference and their clinical picture and laparoscopic cholecystectomy. In our study patients with score 2 and 3 six patients (50\%) had CBD stones and six patients (50\%) didn't have CBD stones .They were subjected to MRCP assessment before sending them to ERCP or LC.

One patient with score 4 was subjected to MRCP assessment before taking up for ERCP. Patient with score 4 had ascending cholangitis in MRCP was later sent for ERCP stenting .Patients (3 patients) with score 5 were taken for ERCP straight and were found to have CBD calculi in $100 \%$ (3patients) of the patients.

Patients with a score of 2, 3 or 4 were made to undergo MRCP to detect $\mathrm{CBD}$ stones, as these patients have a high probability to be having CBD stones but the same is not certain. Instead of subjecting the patients to an unnecessary ERCP, which is quite probable in such cases, it was better to evaluate the biliary tree using MRCP first. If MRCP shows a stone, the patient was asked to undergo stone removal either with ERCP or CBD exploration based on surgeon preference and their clinical picture.

Patients with a score of 5 should directly undergo ERCP, in our data, they have $100 \%$ chance of having a CBD stone and should have urgent decompression of the biliary system to prevent cholangitis. The calculated accuracy shows that the scoring system is an accurate predictor of persistent gallstones, especially for patients with a score of $5(100 \%)$ and comparable accuracy for score $0(88.9 \%)$. The accuracy falls slightly with the addition of patients who score in the middle of the scoring range. This decline in accuracy further supports the clinical implication of having the patient receive an MRCP. MRCP is highly accurate in diagnosing CBD stones (> 90\%). Because ERCP is not without morbidity, MRCP is a good way to select patients for therapeutic ERCP instead of jumping to diagnostic ERCP.

Every patient who underwent ERCP had a CBD stone except for 2 patients one had stricture at common hepatic duct and other patient had to undergo ERCP for Ascending cholangitis which were not picked up on CECT or MRCP .This reduction of negative ERCP rate is important, because ERCP is not without complications. By reducing negative ERCPs, we eliminate the cost of the procedure itself and the cost of treating the complications of ERCP.ERCP along with sphincterotomy, stone extraction, and intraoperative $\mathrm{CBD}$ exploration are 2 ways to remove CBD stones. Both are equally effective in removing $\mathrm{CBD}$ stones, and intraoperative $\mathrm{CBD}$ exploration may have a lesser risk of morbidity, mortality, and recurrence of CBD stones. Performing intraoperative CBD exploration during cholecystectomy instead of a separate ERCP procedure for CBD stone reduces the number of total procedures and length of stay. The decision of choosing the modality for CBD stone removal 
depends on a combination of surgeons' comfort level with intraoperative CBD exploration and the availability of skilled advanced endoscopists at the institution. In our institution one patient with score 2 underwent MRCP assessment and later was taken for LC and was later converted to Open procedure for $\mathrm{CBD}$ exploration due to presence of multiple filling defects on MRCP assessment.

All patients with gallstone pancreatitis are taken for LC after CBD stone removal as definitive treatment for Gall stone pancreatitis. In our institution 36 patients were taken up for LC after CBD evaluation for stones and 2 patients underwent ERCP stenting 1 patients underwent open CBD exploration for stone removal.

\section{Limitations of the Study}

- Smaller sample size than original study

- Shorter study period.

- Limited resources (non availability of intra op cholangiography)

- Selection Bias due to non randomization of sample

\section{Conclusion}

A scoring system has a place in the management of gallstone pancreatitis. The accuracy of the scoring system is $72.5 \%$ in my study. This scoring system helps us to stratify the risk of having retained CBD stones based on a set of their admission laboratory values and initial USG findings. Furthermore, our scoring system and protocol helped to reduce the number of negative ERCPs inour institution. Using this scoring system, we propose the following for patients: those with score 0 or 1 undergo LC with follow up to rule out biliary obstruction or recurrent pancreatitis post- operatively; score 2,3 or 4 undergo MRCP; and score 5 should go directly to ERCP.

In patients who are cholangitic, this scoring system is not applicable because those patients must be treated for their biliary obstruction. In otherwise stable patients, this scoring system can help to stratify the risk of having CBD stones based on set of admission laboratory values, and initial ultrasonographic findings.

From my study I would like to tell that this prospective study is a good step in validating the scoring system. A prospective, large series study is the next step; it will increase the number of subjects and therefore the statistical power. Furthermore, a multicenter evaluation would also take into consideration the varying availability of MRCP, the comfort of the gastroenterologist to perform diagnostic and therapeutic ERCP, and the experience of surgeons to perform intraoperative CBD exploration to make the scoring system and treatment protocol more applicable

\section{References}

1. Norman S, William S P, Ronan o connell and rew W, Mccaskie et al ., The pancreas .Bailey and love's short pratice of surgery" 27th ed.crc press,taylor and francis group 2018;68:1221.1213

2. Yadav D, Lowenfels AB. Trends in the epidemiology of the first attack of acute pancreatitis: a systematic review. Pancreas 2006;33:323-30.

3. Fagenholz PJ, Fern andez-del Castillo C, Harris NS, Pelletier AJ, Camargo CA Jr. Direct medical costs of acute pancreatitis hospitalizations

4. Larson SD, Nealon WH, Evers BM: Management of gallstone pancreatitis. Adv Surg 40:265-284, 2006

5. Brett M, Barker DJ. The world distribution of gallstones. Int J Epidemiol. 1976;5:335

6. Shaffer E. Gallstone disease: Epidemiology of gallbladder stone disease. Best Practice Research Clinical Gastroenterology. 2006;20(6):981-96.

7. Stinton LM, Shaffer EA. Epidemiology of gallbladder disease: Cholelithiasis and cancer. Gut Liver. 2012;6(2):172-187.87

8. $\mathrm{Xu} \mathrm{Q}, \mathrm{Tao} \mathrm{L}, \mathrm{Wu} \mathrm{Q}$, et al. Prevalences of and risk factors for biliary stones and gallbladderpolyps in a large chinese population. HPB. 2012;14(6):373-381 
9. Everhart JE, Khare M, Hill M, Maurer KR. Prevalence and ethnic differences in gall bladder disease in the united states. Gastroenterology. 1999;117(3):632-9.

10. Attili AF, Carulli N, Roda E, et al. Epidemiology of gallstone disease in italy: Prevalence data of the multicenter italian study on cholelithiasis (M.I.COL.). Am J Epidemiol. 1995;141(2):158-165.

11. Barbara L, Sama C, Labate AMM, et al. A population study on the prevalence of gallstone disease: The sirmione study. Hepatology. 1987;7(5):913-917

12. Frossard JL, Steer ML, Pastor CM. Acute pancreatitis. Lancet 2008;371:143-52.

13. van Geenen EJ, van der Peet DL, Bhagirath P, Mulder CJ, Bruno MJ. Etiology and diagnosis of acute biliary pancreatitis. Nat Rev Gastroenterol Hepatol 2010; 7:495-502.

14. Acosta JM, Ledesma CL. Gallstone migration as a cause of acute pancreatitis. N Engl J Med 1974;290:484-7.

15. Telem DA, Bowman K, Hwang J, Chin EH, Nguyen SQ, Divino CM. Selective management of patients with acute biliary pancreatitis. J Gastrointest Surg 2009; $13: 2183-8$

16. Sherman JL, Shi EW, Ranasinghe NE, Sivasankaran MT, Prigoff JG, Divino CM.E rratum: Corrigendum to "Validation and improvement of a proposed scoring system to detect retained common bile duct stones in gallstone pancreatitis" (Surgery (2015) 157(6) (1073-1079) (S0039606015000173)

(10.1016/j.surg.2015.01.005)). Surg (United States). 2016;160(5):1424. doi:10.1016/j.surg.2016.06.001.

\section{Abbreviations}

USG- Ultrasonogram

ERCP- Endoscopic Retrograde Cholangio Pancreaticography

MRCP- Magnetic Resonance Cholangio Pancreaticography

TB-Total Bilirubin

DB- Direct Bilirubin

AP-Alkaline Phosphatase

GGT-Gamma-glutamyl transferase

GB-Gallbladder

CBD-Common bile duct

LC-Laparoscopic Cholecystectomy 